\title{
Retrospective cohort study of trifluridine/ tipiracil (TAS-102) plus bevacizumab versus trifluridine/tipiracil monotherapy for metastatic colorectal cancer
}

Daisuke Kotani ${ }^{1}$, Yasutoshi Kuboki ${ }^{1,2^{*}}$, Satoshi Horasawa ${ }^{1,3}$, Asumi Kaneko ${ }^{4}$, Yoshiaki Nakamura ${ }^{1,3}$, Akihito Kawazoe ${ }^{1}$, Hideaki Bando ${ }^{1,5}$, Hiroya Taniguchi ${ }^{1,3}$, Kohei Shitara', Takashi Kojima' ${ }^{1}$, Akihito Tsuji ${ }^{6}$ and Takayuki Yoshino ${ }^{1}$

\begin{abstract}
Background: A previous phase I/II C-TASK FORCE study of trifluridine/tipiracil plus bevacizumab for patients with heavily pretreated metastatic colorectal cancer $(\mathrm{mCRC})$ showed promising activity with an acceptable toxicity profile. This retrospective study aimed to investigate the safety and efficacy of trifluridine/tipiracil plus bevacizumab compared with trifluridine/tipiracil monotherapy in patients with heavily pretreated $m C R C$ in clinical settings.

Methods: Records of patients with mCRC refractory to standard therapies who initiated trifluridine/tipiracil plus bevacizumab from January 2016 to March 2018 or trifluridine/tipiracil monotherapy from June 2014 to December 2015 were retrospectively reviewed at our institution.

Results: Totally, 60 patients received trifluridine/tipiracil plus bevacizumab and 66 received trifluridine/tipiracil monotherapy. All patients had previously received standard chemotherapy. Median progression-free survival (PFS) was 3.7 months [95\% confidence interval $(\mathrm{Cl}), 2.3-5.1]$ in the trifluridine/tipiracil plus bevacizumab group and 2.2 months $(95 \% \mathrm{Cl}, 1.8-2.6)$ in the trifluridine/tipiracil monotherapy group [hazards ratio (HR) 0.69 ; $95 \% \mathrm{Cl} 0.48-0.99$ ]. PFS rate at 16 weeks was $46.6 \%$ for the trifluridine/tipiracil plus bevacizumab group and $33.9 \%$ for the trifluridine/ tipiracil monotherapy group. Although a relatively higher incidence of grade $\geq 3$ neutropenia was observed in the trifluridine/tipiracil plus bevacizumab group than that in the other group ( $50.0 \%$ vs. $40.9 \%, p=0.371)$, the incidence of febrile neutropenia was not high (3.3\% vs. $7.8 \%, p=0.444)$.
\end{abstract}

Conclusions: In real-world settings, trifluridine/tipiracil plus bevacizumab prolonged PFS and helped achieve higher 16-week PFS rate compared with trifluridine/tipiracil monotherapy in patients with heavily pretreated mCRC with manageable toxicities.

Trial registration: Retrospectively registered.

Keywords: Trifluridine/tipiracil plus bevacizumab, TAS-102, mCRC, Lonsurf

\footnotetext{
* Correspondence: ykuboki@east.ncc.go.jp

${ }^{1}$ Department of Gastrointestinal Oncology, National Cancer Center Hospital

East, Kashiwa, Japan

${ }^{2}$ Department of Experimental Therapeutics, National Cancer Center Hospital

East, 6-5-1 Kashiwanoha, Kashiwa, Chiba 277-8577, Japan

Full list of author information is available at the end of the article
}

(c) The Author(s). 2019 Open Access This article is distributed under the terms of the Creative Commons Attribution 4.0 International License (http://creativecommons.org/licenses/by/4.0/), which permits unrestricted use, distribution, and reproduction in any medium, provided you give appropriate credit to the original author(s) and the source, provide a link to the Creative Commons license, and indicate if changes were made. The Creative Commons Public Domain Dedication waiver (http://creativecommons.org/publicdomain/zero/1.0/) applies to the data made available in this article, unless otherwise stated. 


\section{Background}

Colorectal cancer (CRC) is the second leading cause of cancer-related death worldwide [1]. The development of combination chemotherapy regimens comprising cytotoxic agents (e.g., fluoropyrimidine, oxaliplatin, and irinotecan) and molecular targeted therapies (e.g., bevacizumab, ramucirumab, ziv-aflibercept, cetuximab, panitumumab, and regorafenib) have increased the survival of patients with metastatic CRC (mCRC) by approximately 30 months [2-7].

Trifluridine/tipiracil (TAS-102) is a novel, oral combination comprising the thymidine-based nucleoside analog trifluridine and tipiracil hydrochloride at a molar ratio of 1:0.5. Trifluridine is incorporated into DNA after phosphorylation by thymidine kinase 1 (TK1). We previously reported results from a randomized phase 2 study of trifluridine/tipiracil (J00310040030), and this therapy was first approved in Japan in 2014 [8]. More recently, the international phase 3 RECOURSE trial has demonstrated a more significant overall survival (OS) benefit of trifluridine/ tipiracil compared with placebo, with acceptable toxicity, in patients with refractory mCRC [7]. In addition, the Asian phase 3 TERRA trial has reported the survival benefit and safety of trifluridine/tipiracil in Asian population [9] Based on these findings, trifluridine/tipiracil has been approved by many countries and regions including the US Food and Drug Administration and European Medicines Agency.

The combination of trifluridine/tipiracil plus bevacizumab has been demonstrated to have synergistic activity in a xenograft model of human CRC [10]. We have reported results from the phase $1 / 2$ C-TASK FORCE showing a promising activity of the aforementioned combination in patients with pretreated mCRC. The primary endpoint of 16-week progression-free survival (PFS) was $42.9 \%$ [80\% confidence interval (CI), 27.8-59.0]. The most common grade $\geq 3$ adverse events were neutropenia (72\%), leucopenia (44\%), anemia (16\%), febrile neutropenia (16\%), and thrombocytopenia (12\%) [11]. Managing the higher incidence of hematological toxicities is crucial to reduce the risks of serious treatment-related adverse events and maximize the efficacy of trifluridine/tipiracil plus bevacizumab treatment. Furthermore, the non-comparative phase 2 TASCO1 study evaluated the efficacy and safety of trifluridine/tipiracil plus bevacizumab and capecitabine plus bevacizumab and provided evidence demonstrating the efficacy of trifluridine/tipiracil plus bevacizumab in patients with untreated mCRC who were not eligible for standard first-line intensive therapy. The primary endpoint of PFS was 9.2 months in the trifluridine/tipiracil plus bevacizumab group and 7.8 months in the capecitabine plus bevacizumab group [12].
Although trifluridine/tipiracil plus bevacizumab is a promising regimen for mCRC patients, little is known about the survival benefit and safety profile of the combination compared with trifluridine/tipiracil monotherapy. Therefore, the aim of this study was to investigate efficacy and safety of trifluridine/tipiracil plus bevacizumab compared with trifluridine/tipiracil monotherapy in the clinical practice.

\section{Methods}

\section{Study design and patients}

Clinical data of patients with $\mathrm{mCRC}$ who received trifluridine/tipiracil plus bevacizumab or trifluridine/tipiracil monotherapy at the National Cancer Center Hospital East was retrospectively collected. Study protocol was approved by the institutional review board. Informed consent requirement was waived due to the study's observational retrospective design, with an opt-out opportunity provided at the institution's website. Patient follow-up was performed until December 2018.

Eligibility criteria were as follows: histologically confirmed colorectal adenocarcinoma; no prior treatment with regorafenib; refractory or intolerant to fluoropyrimidine, oxaliplatin, and irinotecan, regardless of angiogenesis inhibitors or anti-EGFR antibody (if $R A S$ wildtype); Eastern Cooperative Oncology Group performance status (ECOG PS) 0 to 2; adequate organ function; concurrent treatment with trifluridine/tipiracil plus bevacizumab from January 2016 to March 2018 or trifluridine/tipiracil monotherapy from June 2014 to December 2015.

\section{Study procedures}

Trifluridine/tipiracil plus bevacizumab regimen consisted of trifluridine/tipiracil $35 \mathrm{mg} / \mathrm{m}^{2}$ of body surface area, given orally twice a day on days $1-5$ and 8-12 in a 28-day cycle, and bevacizumab $5 \mathrm{mg} / \mathrm{kg}$ of bodyweight, administered by intravenous infusion every 2 weeks. Trifluridine/tipiracil monotherapy consisted of trifluridine/ tipiracil $35 \mathrm{mg} / \mathrm{m}^{2}$ of body surface area, given orally twice a day on days $1-5$ and 8-12 in a 28-day cycle.

The following baseline characteristics were collected for each patient: age, gender, ECOG PS, primary tumor location, history of primary resection, number of metastatic organs, time from first-line chemotherapy start, time from prior bevacizumab, prior chemotherapy agents, RAS status (KRAS exons 2, 3, and 4 and NRAS exons 2, 3, and 4), BRAF V600E mutation status, and microsatellite instability (MSI) status, if available.

\section{Outcomes}

Efficacy endpoints included PFS, defined as time from study treatment initiation to disease progression or death due to any cause; OS, defined as time from study 
treatment start to death from any cause; overall response rate (ORR), defined as proportion of patients with a complete or partial response to study treatment; disease control rate (DCR), defined as proportion of patients with a complete or partial response plus stable disease lasting more than 6 weeks from study treatment start. Tumor response was assessed by investigators using the Response Evaluation Criteria in Solid Tumors version 1.1. Adverse events were evaluated using the Common Terminology Criteria for Adverse Events version 4.03.

\section{Statistical analysis}

PFS and OS were compared between treatment groups using log-rank test with a two-sided significance level of $p=0.05$. Hazard ratio (HR) and corresponding 95\% CI were determined using a Cox proportional hazard model. Survival curves were generated using Kaplan-Meier estimates. Univariate and multivariate analyses were performed to evaluate the impact of trifluridine/tipiracil plus bevacizumab or trifluridine/tipiracil monotherapy treatments. Regression analysis covariates included treatment group, age, gender, ECOG PS, primary tumor location, RAS status, time from first-line chemotherapy start, and time from prior bevacizumab. Multivariate Cox analysis was employed using forward stepwise regression. Enter and remove limits were $p=0.05$ and $p=0.20$, respectively. Confounding variables considered in multivariate analysis included age $(<65$ years old vs. $\geq 65$ years old), gender (male vs. female), ECOG PS (0 vs. $\geq 1$ ), primary tumor location (right vs. left), $R A S$ status (wild-type vs. mutant), time from first-line chemotherapy start ( $\geq 18$ months vs. $<18$ months), and time from prior bevacizumab $(\leq 1$ month vs. $>1$ month or no prior bevacizumab). ORR, DCR, and safety analyses between treatment groups were performed using Fisher's exact test. Follow-up time was defined as time from study treatment start until the last followup date for censored cases. Statistical analyses were performed using IBM SPSS statistics version 22.0 (IBM Corp, Armonk, NY), and two-sided $p<0.05$ was considered statistically significant.

\section{Results}

\section{Patients}

Sixty patients received trifluridine/tipiracil plus bevacizumab and 66 patients received trifluridine/tipiracil monotherapy. Patient demographics and baseline characteristics are shown in Table 1. All patients had previously received fluoropyrimidine, oxaliplatin, and irinotecan. Most of the patients in each group had a history of treatment with angiogenesis inhibitors, including bevacizumab, ramucirumab, or ziv-aflibercept. Approximately half of patients had $R A S$ wild-type tumors, and no patient had MSI-high tumor. BRAF V600E mutation was detected in one patient (1.7\%) in the trifluridine/ tipiracil plus bevacizumab group and in four patients (6.1\%) in the trifluridine/tipiracil monotherapy group. Patients with left-sided primary tumors were dominant in both groups and comprised $81.7 \%$ of the trifluridine/tipiracil plus bevacizumab group and $84.8 \%$ of the trifluridine/tipiracil monotherapy group. Median interval from study treatment start to first computed tomography evaluation was 1.8 months in both groups. Median follow-up was 7.1 months in the trifluridine/tipiracil plus bevacizumab groups and 7.2 months in the trifluridine/tipiracil monotherapy group. After study treatment discontinuation, $41.7 \%$ of patients in the trifluridine/tipiracil plus bevacizumab groups and $48.5 \%$ of patients in the trifluridine/ tipiracil monotherapy group received subsequent antitumor therapy including regorafenib (31.7 vs. 39.4\%), clinical trial therapy (6.7 vs. $4.5 \%)$, and cytotoxic chemotherapy (3.3 vs. $6.0 \%)$.

\section{Efficacy}

Patients in the trifluridine/tipiracil plus bevacizumab group had significantly longer PFS compared with those in the trifluridine/tipiracil monotherapy group (HR 0.69; 95\% CI 0.48-0.99; log-rank $p=0.037$ ). Median PFS was 3.7 months (95\% CI 2.3-5.1 months) in the trifluridine/ tipiracil plus bevacizumab group and 2.2 months (95\% CI 1.8-2.6 months) in the trifluridine/tipiracil monotherapy group (Fig. 1a). PFS rate at 16 weeks was 46.6 and $33.9 \%$, respectively. In multivariate analysis, similar PFS was observed between groups (adjusted HR 0.62; 95\% CI $0.42-0.90, p=0.01)$. Subgroup analyses also showed a PFS benefit for trifluridine/tipiracil plus bevacizumab compared with trifluridine/tipiracil monotherapy for all parameters except ECOG PS (Table 2).

Median OS was 8.6 months (95\% CI 6.9-10.3 months) for trifluridine/tipiracil plus bevacizumab and 8.0 months (95\% CI 6.6-9.4 months) for trifluridine/tipiracil monotherapy (Fig. 1b). In multivariate analysis, an OS benefit was also observed for trifluridine/tipiracil plus bevacizumab compared with trifluridine/tipiracil monotherapy, but without statistical significance (HR 0.74; 95\% CI $0.48-$ 1.14; $\log$-rank $p=0.164$ ). Similarly to PFS, longer OS was observed for all subgroups, except ECOG PS.

Three patients in the trifluridine/tipiracil plus bevacizumab group and one patient in the trifluridine/tipiracil monotherapy group had partial response, resulting in a 5.0 and $1.5 \%$ ORR for each group, respectively $(p=0.35)$. Disease control was achieved in 32 patients $(53.3 \%)$ in the trifluridine/tipiracil plus bevacizumab group and in 30 patients $(45.5 \%)$ in the trifluridine/tipiracil monotherapy group $(p=0.48)$ (Table 3$)$. Additionally, proportion of patients with 6 months or longer of disease control 
Table 1 Patient characteristics

\begin{tabular}{|c|c|c|c|c|c|}
\hline & & $\begin{array}{l}\text { Trifluridine } \\
\text { bevacizum }\end{array}$ & $\begin{array}{l}\text { il plus } \\
\text { up }\end{array}$ & $\begin{array}{l}\text { Trifluridine/t } \\
\text { group }\end{array}$ & notherapy \\
\hline & & $N=60$ & $\%$ & $N=66$ & $\%$ \\
\hline \multirow[t]{2}{*}{ Age } & Median (range) & $60(23-79)$ & & $65(30-80)$ & \\
\hline & $\geq 65$ years old & 19 & 31.7 & 34 & 51.5 \\
\hline Gender & Male & 35 & 58.3 & 42 & 63.6 \\
\hline \multirow[t]{3}{*}{ ECOG PS } & 0 & 35 & 58.3 & 42 & 63.6 \\
\hline & 1 & 24 & 40.0 & 21 & 31.8 \\
\hline & 2 & 1 & 1.7 & 3 & 4.5 \\
\hline \multirow[t]{2}{*}{ Primary location } & Right & 11 & 18.3 & 10 & 15.2 \\
\hline & Left & 49 & 81.7 & 56 & 84.8 \\
\hline \multirow[t]{3}{*}{ Number of metastatic organs } & 1 & 6 & 10.0 & 13 & 19.7 \\
\hline & 2 & 26 & 43.3 & 39 & 59.1 \\
\hline & $\geq 3$ & 28 & 46.7 & 14 & 21.2 \\
\hline \multirow[t]{2}{*}{ Time from start of first-line chemotherapy } & $<18$ months & 22 & 36.7 & 23 & 34.8 \\
\hline & $\geq 18$ months & 38 & 63.3 & 43 & 65.2 \\
\hline \multirow[t]{2}{*}{ Time from prior bevacizumab } & $\leq 1$ month & 34 & 56.7 & 33 & 50.0 \\
\hline & $>1$ month or no prior bevacizumab & 26 & 43.3 & 33 & 50.0 \\
\hline \multirow[t]{4}{*}{ Number of prior regimens } & 1 & 4 & 6.7 & 2 & 3.0 \\
\hline & 2 & 29 & 48.3 & 33 & 50.0 \\
\hline & 3 & 15 & 25.0 & 16 & 24.2 \\
\hline & $\geq 4$ & 12 & 20.0 & 15 & 22.7 \\
\hline \multirow[t]{5}{*}{ Prior chemotherapy agents } & Fluoropyrimidine & 60 & 100 & 66 & 100 \\
\hline & Irinotecan & 60 & 100 & 66 & 100 \\
\hline & Oxaliplatin & 60 & 100 & 66 & 100 \\
\hline & Angiogenesis inhibitors & 58 & 96.7 & 61 & 92.4 \\
\hline & Anti-EGFR antibodies & 27 & 45.0 & 27 & 40.9 \\
\hline \multirow[t]{2}{*}{ RAS status } & Wild-type & 28 & 46.7 & 30 & 45.5 \\
\hline & Mutant & 32 & 53.3 & 36 & 54.5 \\
\hline \multirow[t]{4}{*}{ BRAF status } & Wild-type & 52 & 86.7 & 52 & 78.8 \\
\hline & V600E mutant & 1 & 1.7 & 4 & 6.1 \\
\hline & Non-V600E mutant & 2 & 3.3 & 0 & 0 \\
\hline & Unknown & 5 & 8.3 & 10 & 15.2 \\
\hline \multirow[t]{2}{*}{ MSI status } & MSS & 53 & 88.3 & 51 & 77.3 \\
\hline & Unknown & 7 & 11.7 & 15 & 22.7 \\
\hline
\end{tabular}

ECOG PS Eastern Cooperative Oncology Group performance status, EGFR epidermal growth factor receptor, MSS microsatellite stable

were significantly higher in the trifluridine/tipiracil plus bevacizumab group than the trifluridine/tipiracil monotherapy group ( $26.7 \%$ vs. $12.1 \%, p=0.04)$.

\section{Safety}

All patients initially received full-dose trifluridine/tipiracil and bevacizumab or trifluridine/tipiracil monotherapy. Any cycle delay for $\geq 4$ days was registered in 37 (61.7\%) and 27 (40.9\%) patients in the trifluridine/tipiracil plus bevacizumab group and trifluridine/tipiracil monotherapy groups $(p=0.02)$, and trifluridine/tipiracil dose reductions were required in $10(16.7 \%)$ and 15 $(22.7 \%)$ patients $(p=0.50)$, respectively.

Adverse events are summarized in Table 4. Overall, grade $\geq 3$ adverse events were more frequent in the trifluridine/tipiracil plus bevacizumab than in the trifluridine/tipiracil monotherapy group $(n=41,68.3 \%$ vs. $n=$ $36,54.5 \% ; p=0.14$ ). Incidence of grade $\geq 3$ neutropenia was slightly higher in the trifluridine/tipiracil plus bevacizumab group than in the trifluridine/tipiracil monotherapy group, although this difference was not statistically significant $(50.0 \%$ vs. $40.9 \% ; p=0.37)$. In 
A.

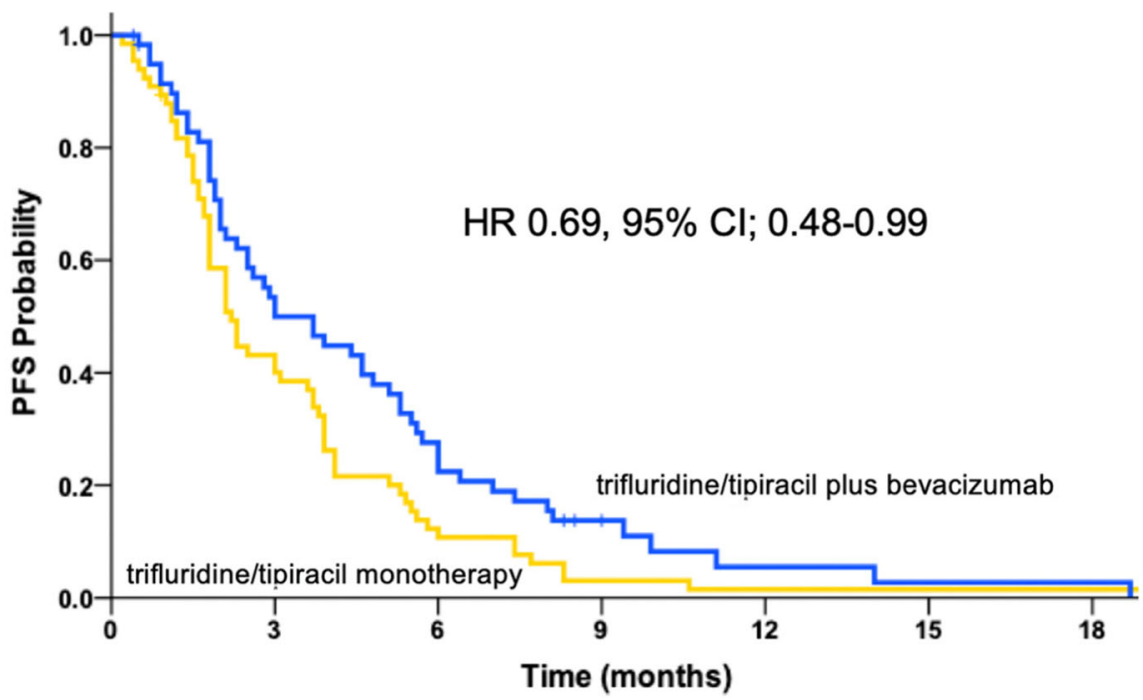

Number at risk

Trifluridine/tipiracil plus bevacizumab 60 $60 \quad 31$

Trifluridine/tipiracil

66

\section{8}

16

6

2

1

1

B.

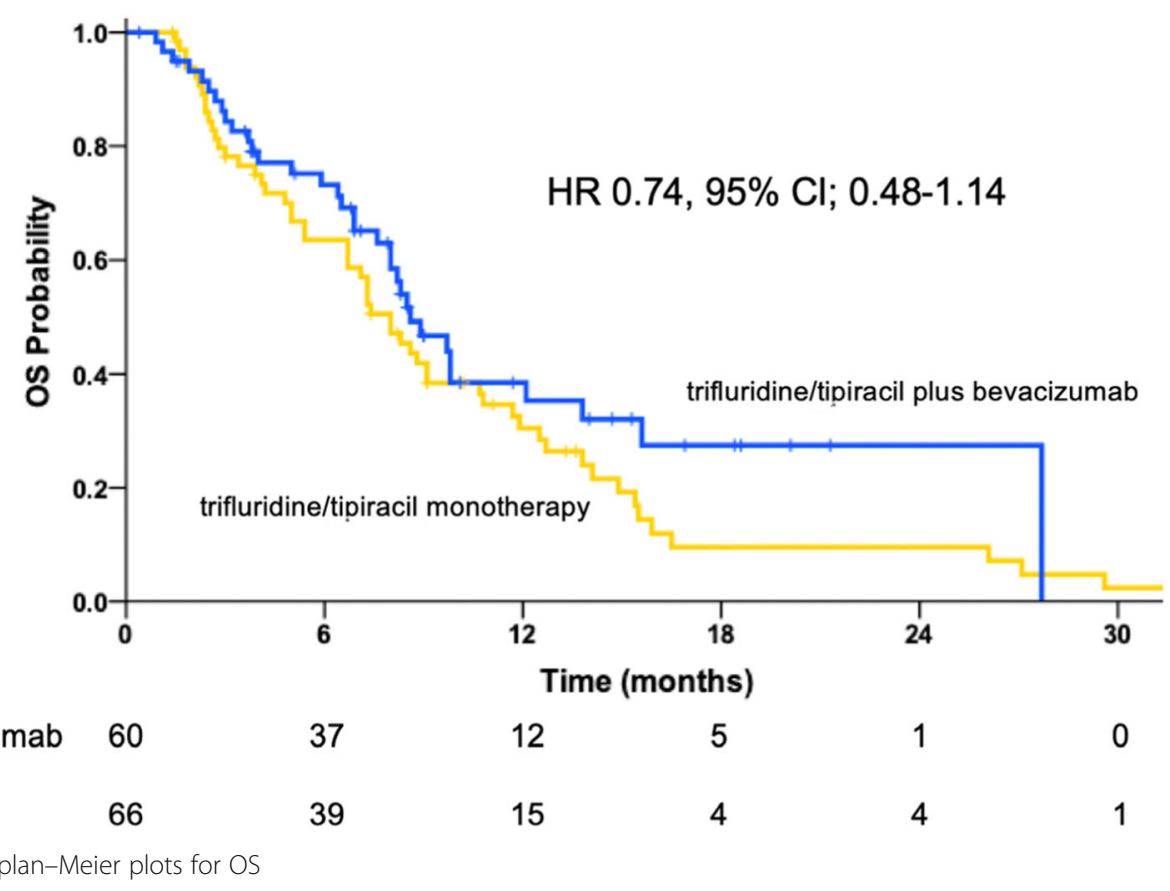

contrast, no increased incidence of febrile neutropenia was observed in the trifluridine/tipiracil plus bevacizumab compared with the trifluridine/tipiracil monotherapy group (3.3\% vs. $7.8 \%$; $p=0.444)$. Ten patients $(16.7 \%)$ in the trifluridine/tipiracil plus bevacizumab group and four patients (6.1\%) in the trifluridine/tipiracil monotherapy group received granulocyte colony-stimulating factor (G-CSF), with no G-CSF prophylaxis use in the both groups. Any grade proteinuria $(41.7 \%$ vs. $13.6 \%$; $p<0.01)$ and hypertension $(38.3 \%$ vs. $16.7 \% ; p<0.01)$, potentially associated with bevacizumab, were more common in the trifluridine/tipiracil plus bevacizumab group. Emergency hospital admission 
Table 2 PFS and OS subgroup analyses

\begin{tabular}{|c|c|c|c|c|c|c|c|c|}
\hline & & \multirow[b]{2}{*}{$\mathrm{N}$} & \multicolumn{3}{|l|}{ PFS } & \multicolumn{3}{|l|}{ OS } \\
\hline & & & $\mathrm{HR}$ & $95 \% \mathrm{Cl}$ & Interaction $p$ & $\mathrm{HR}$ & $95 \% \mathrm{Cl}$ & Interaction $\mathrm{p}$ \\
\hline \multicolumn{2}{|l|}{ All patients } & 126 & 0.69 & $0.48-0.99$ & & 0.74 & $0.48-1.14$ & \\
\hline \multirow[t]{2}{*}{ Age } & $<65$ years old & 73 & 0.53 & $0.31-0.88$ & 0.542 & 0.85 & $0.48-1.50$ & 0.300 \\
\hline & $\geq 65$ years old & 53 & 0.74 & $0.41-1.32$ & & 0.49 & $0.23-1.08$ & \\
\hline \multirow[t]{2}{*}{ Gender } & Male & 77 & 0.72 & $0.45-1.15$ & 0.560 & 0.98 & $0.57-1.70$ & 0.162 \\
\hline & Female & 49 & 0.61 & $0.34-1.09$ & & 0.51 & $0.25-1.04$ & \\
\hline \multirow[t]{2}{*}{ ECOG PS } & 0 & 77 & 0.50 & $0.31-0.80$ & 0.009 & 0.54 & $0.30-0.99$ & 0.033 \\
\hline & $\geq 1$ & 49 & 1.42 & $0.79-2.55$ & & 1.46 & $0.76-2.82$ & \\
\hline \multirow[t]{2}{*}{ Primary location } & Left & 105 & 0.65 & $0.44-0.98$ & 0.758 & 0.72 & $0.44-1.16$ & 0.986 \\
\hline & Right & 21 & 0.64 & $0.26-1.59$ & & 0.62 & $0.21-1.80$ & \\
\hline \multirow{2}{*}{$\begin{array}{l}\text { Time from start of first-line } \\
\text { chemotherapy }\end{array}$} & $\geq 18$ months & 81 & 0.71 & $0.45-1.11$ & 0.359 & 0.66 & $0.38-1.16$ & 0.635 \\
\hline & $<18$ months & 45 & 0.67 & $0.36-1.25$ & & 0.78 & $0.39-1.55$ & \\
\hline \multirow[t]{2}{*}{ Time from prior bevacizumab } & $\leq 1$ month & 67 & 0.48 & $0.28-0.82$ & 0.165 & 0.64 & $0.37-1.12$ & 0.975 \\
\hline & $>1$ month or no prior bevacizumab & 59 & 0.77 & $0.45-1.33$ & & 0.73 & $0.36-1.45$ & \\
\hline \multirow[t]{2}{*}{ RAS status } & Wild-type & 58 & 0.87 & $0.50-1.49$ & 0.147 & 0.67 & $0.35-1.28$ & 0.580 \\
\hline & Mutant & 68 & 0.52 & $0.31-0.87$ & & 0.79 & $0.44-1.41$ & \\
\hline \multirow[t]{2}{*}{ History of bevacizumab } & yes & 118 & 0.75 & $0.51-1.09$ & 0.47 & 0.70 & $0.45-1.09$ & 0.88 \\
\hline & no & 8 & 0.50 & $0.09-2.67$ & & 3.30 & $0.29-37.7$ & \\
\hline
\end{tabular}

Cl confidence interval, ECOG PS Eastern Cooperative Oncology Group performance status, HR hazard ratio, OS overall survival, PFS progression-free survival

was required for $15(25.0 \%)$ and $19(28.8 \%)$ patients in the trifluridine/tipiracil plus bevacizumab and trifluridine/tipiracil monotherapy groups, respectively. No treatmentrelated deaths occurred in the both groups.

\section{Discussion}

Although the clinical evidence for trifluridine/tipiracil plus bevacizumab in pretreated mCRC patients only comes from a phase 1/2 C-TASK FORCE trial with a single-arm design, the present study showed clinical benefit with manageable toxicities for this combination in the real-world setting.

The PFS improvement associated with trifluridine/tipiracil plus bevacizumab can be regarded as clinically meaningful in this patient population. Considering the median PFS difference between trifluridine/tipiracil and placebo of 0.3 and 0.2 months reported in RECOURSE and TERRA trials, respectively, the absolute 1.5 -month median PFS improvement observed in this study is clinically meaningful in salvage setting. Of note, a higher 16-week PFS rate was observed with trifluridine/tipiracil plus bevacizumab compared with trifluridine/tipiracil monotherapy (46.6 vs. $33.9 \%$ ), consistent with the primary endpoint of the CTASK FORCE trial. Furthermore, although limited efficacy was reported in the C-TASK FORCE trial for trifluridine/ tipiracil plus bevacizumab in patients with mutant $R A S$, a consistent benefit of the combination was observed in subgroup analyses in this study, irrespectively of RAS status. Although not statistically significant, a 0.74 HR for OS was observed in favor of trifluridine/tipiracil plus bevacizumab in this study. Fewer OS events in the trifluridine/tipiracil plus bevacizumab group due to limited follow-up may have failed to show statistical difference between both groups. In subgroup analyses, patients with ECOG PS 0 especially

Table 3 Overall response

\begin{tabular}{|c|c|c|c|c|c|}
\hline \multirow{2}{*}{$\begin{array}{l}\text { Best } \\
\text { response }\end{array}$} & \multicolumn{2}{|c|}{ Trifluridine/tipiracil plus bevacizumab group } & \multicolumn{2}{|c|}{ Trifluridine/tipiracil monotherapy group } & \multirow[t]{2}{*}{$P$} \\
\hline & $N=60$ & $\%$ & $N=66$ & $\%$ & \\
\hline $\mathrm{PR}$ & 3 & 5.0 & 1 & 1.5 & \\
\hline SD & 29 & 48.3 & 29 & 43.9 & \\
\hline PD & 25 & 41.7 & 34 & 51.5 & \\
\hline $\mathrm{NE}$ & 3 & 5.0 & 2 & 3.0 & \\
\hline ORR & 3 & 5.0 & 1 & 1.5 & 0.346 \\
\hline DCR & 32 & 53.3 & 30 & 45.5 & 0.476 \\
\hline
\end{tabular}

$D C R$ disease control rate, $N E$ not evaluated, $O R R$ overall response rate, $P D$ progressive disease, $P R$ partial response, $S D$ stable disease 
Table 4 Adverse events

\begin{tabular}{|c|c|c|c|c|c|c|}
\hline & \multicolumn{3}{|c|}{ Trifluridine/tipiracil plus bevacizumab group $(N=60)$} & \multicolumn{3}{|c|}{ Trifluridine/tipiracil monotherapy group $(N=66)$} \\
\hline & Any grade (\%) & Grade $3(\%)$ & Grade $4(\%)$ & Any grade (\%) & Grade $3(\%)$ & Grade $4(\%)$ \\
\hline \multicolumn{7}{|l|}{ Hematological } \\
\hline Neutropenia & $41(68.3)$ & $25(41.7)$ & $5(8.3)$ & $47(71.2)$ & $19(28.8)$ & $8(12.1)$ \\
\hline Leucopenia & $49(81.7)$ & $23(38.3)$ & $0(0)$ & $47(71.2)$ & $17(25.8)$ & $2(3.0)$ \\
\hline Anemia & $52(86.7)$ & $8(13.3)$ & $1(1.7)$ & $60(90.9)$ & $12(18.2)$ & $2(3.0)$ \\
\hline Thrombocytopenia & $26(43.3)$ & $1(1.7)$ & $1(1.7)$ & $24(36.4)$ & $2(3.0)$ & $0(0)$ \\
\hline \multicolumn{7}{|l|}{ Non-hematological } \\
\hline Proteinuria & $25(41.7)$ & $4(6.7)$ & $0(0)$ & $9(13.6)$ & $1(1.5)$ & $0(0)$ \\
\hline Hypertension & $23(38.3)$ & $4(6.7)$ & $0(0)$ & $11(16.7)$ & $0(0)$ & $0(0)$ \\
\hline Febrile neutropenia & $2(3.3)$ & $2(3.3)$ & $0(0)$ & $5(7.8)$ & $5(7.8)$ & $0(0)$ \\
\hline Gastrointestinal perforation & $2(3.3)$ & $2(2.9)$ & $0(0)$ & $1(1.5)$ & $1(1.5)$ & $0(0)$ \\
\hline Fatigue & $30(50.0)$ & $0(0)$ & $0(0)$ & $25(37.9)$ & $0(0)$ & $0(0)$ \\
\hline Anorexia & $25(41.7)$ & $0(0)$ & $0(0)$ & $27(40.9)$ & $1(1.5)$ & $0(0)$ \\
\hline Nausea & $10(16.7)$ & $0(0)$ & $0(0)$ & $15(22.7)$ & $0(0)$ & $0(0)$ \\
\hline Diarrhea & $5(8.3)$ & $0(0)$ & $0(0)$ & $8(12.1)$ & $0(0)$ & $0(0)$ \\
\hline Vomiting & $2(3.3)$ & $0(0)$ & $0(0)$ & $4(6.1)$ & $0(0)$ & $0(0)$ \\
\hline
\end{tabular}

benefited from trifluridine/tipiracil plus bevacizumab in both PFS and OS. Although no clear benefit of trifluridine/ tipiracil plus bevacizumab were observed in patients with $\geq$ ECOG PS 1, it is not sufficient reason to refrain from using trifluridine/tipiracil plus bevacizumab in those patients, considering retrospective nature of this study.

Trifluridine/tipiracil plus bevacizumab toxicities were well tolerated in the clinical practice setting. Frequency of grade $\geq 3$ neutropenia in the trifluridine/ tipiracil plus bevacizumab group in this study was lower than in the C-TASK FORCE trial (50.0 vs. $72 \%)$. Along with a relatively lower frequency of grade $\geq 3$ neutropenia, G-CSF was used in $16.8 \%$ of patients in the trifluridine/tipiracil plus bevacizumab group in this study compared with $28 \%$ of patients in the C-TASK FORCE trial. However, considering the common occurrence of grade $\geq 3$ neutropenia, the higher proportion of cycle initiation delay in the trifluridine/tipiracil plus bevacizumab group, and the patient population in salvage setting, appropriate supportive intervention with G-CSF or temporary dose interruptions were still important for safety management with trifluridine/tipiracil plus bevacizumab. As bevacizumab - related toxicities, incidence of any grade proteinuria and hypertension were significantly higher in the trifluridine/tipiracil plus bevacizumab group. In the salvage line setting, since almost all patients have received one or more prior angiogenesis inhibitor including bevacizumab, these bevacizumab - related toxicities should be monitored carefully. Notably, such safety management was not associated with an increase in emergency hospital admissions with trifluridine/tipiracil plus bevacizumab, and the incidence of serious adverse events in this study was similar to the observed in RECOURSE and C-TASK FORCE trials and in clinical practice, as previously reported from our institution [13].

Efficacy and safety of trifluridine/tipiracil plus bevacizu$\mathrm{mab}$ is also being investigated in prospective clinical trials. The phase 2 JFMC51-1702-C7 study (UMIN000030077) will validate the use of trifluridine/tipiracil plus bevacizumab in pretreated mCRC patients. Furthermore, this combination is an effective (ORR 40.5\% and DCR 86.5\%) and well-tolerated first-line treatment regimen for elderly patients (KSCC1602; UMIN000025241) [14]. More recently, a randomized Danish study of 80 patients with heavily pretreated $\mathrm{mCRC}$ reported a PFS extension with trifluridine/tipiracil plus bevacizumab compared with trifluridine/tipiracil monotherapy [15]. Based on the abovementioned findings, several phase 2 or 3 studies are in place, including studies investigating this regimen as postinduction chemotherapy maintenance (ALEXANDRIA; NCT02654639) and as second-line treatment for mCRC patients who failed first-line oxaliplatin-based chemotherapy versus FOLFIRI (or S-1 plus irinotecan) plus bevacizumab (TRUSTY; JapicCTI-173,618) [16].

This study has several limitations. Firstly, as previously noted, it was a non-randomized retrospective study in a single institution with a limited sample size. Secondly, no patients received prior regorafenib. However, the CTASK FORCE trial also did not allow prior regorafenib and the study population was very similar to the Japanese subset of the RECOURSE trial. Finally, all patients enrolled in this study were Japanese. The absence of 
ethnic differences in the RECOURSE and TERRA trials could enable results to be applied to all patients, regardless of race.

\section{Conclusions}

In conclusion, in the present study trifluridine/tipiracil plus bevacizumab was shown to have superior clinical activity compared with trifluridine/tipiracil monotherapy in patients with heavily pretreated mCRC. Additionally, similarly to trifluridine/tipiracil monotherapy, toxicities observed with the combination were manageable in the real-world setting.

\section{Abbreviations \\ Cl: Confidence interval; CRC: Colorectal cancer; DCR: Disease control rate; ECOG PS: Eastern Cooperative Oncology Group performance status; G- CSF: Granulocyte colony-stimulating factor; HR: Hazards ratio: mCRC: Metastatic colorectal cancer; MSI: Microsatellite instability; ORR: Overall response rate; OS: Overall survival; PFS: Progression-free survival; TK1: Thymidine kinase 1 \\ Acknowledgements \\ Not applicable. \\ Authors' contributions \\ DK initiated the study, participated in its design and data collection, performed statistical analyses, and drafted the manuscript. YK was responsible for study conception and helped manuscript writing. SH and AK1 participated in data collection. YN, AK2, HB, HT, KS, TK, AT edited manuscript. TY helped manuscript writing and participated in manuscript editing. All authors read and approved the final manuscript.}

\section{Funding}

This research did not receive any specific grant from funding agencies in the public, commercial, or not-for-profit sectors.

\section{Availability of data and materials}

Not applicable.

\section{Ethics approval and consent to participate}

Study protocol was approved by the institutional review board (National Cancer Center IRB, ref. 2017-279). Informed consent requirement was waived due to the study's observational retrospective design, with an opt-out opportunity provided at the institution's website.

\section{Consent for publication}

Not applicable.

\section{Competing interests}

DK reports receiving honoraria from Takeda, Chugai, Lilly, and Merck Serono. YK reports receiving honoraria from Taiho, research funding from Taiho, Takeda, Ono, AstraZeneca, INC research, Daiichi-Sankyo, and Boehringer Ingelheim. YN reports research funding from Ono and Taiho. AK2 reports receiving research funding from Ono, Sumitomo Dainippon and Taiho. HB reports receiving lecturer fee from Taiho and Lilly; research expenses from Taiho, AstraZeneca, and Sysmex. HT reports receiving honoraria from Takeda, Chugai, Merck Serono, Taiho, Bayer, Lilly, Yakult, and Sanofi. KS reports paid consulting or advisory roles for Astellas, Lilly, Bristol-Myers Squibb, Takeda, Pfizer, Ono and MSD; honoraria from Novartis, AbbVie, and Yakult; and research funding from Astellas, Lilly, Ono, Sumoitomo Dainippon, Daiichi Sankyo, Taiho, Chugai, MSD and Medi Science. TK reports receiving research funding from Shionogi, MSD, Ono Pharmaceutical, Chugai, Oncolys BioPharma, Astellas Amgen BioPharma, and Parexel. AT reports receiving honoraria from Daiichi-Sankyo, Taiho, Chugai, Merck Serono, Takeda, and Bristol-Myers Squibb; speaker's bureau from Chugai, Taiho, Takeda, and Merck Serono. YK reports receiving honoraria Takeda, Chugai, Bristol-Myers Squibb, Ono, Merck Biopharma, Taiho, Bayer, Lilly, Yakult Honsha, Sanofi, Nipro, Moroo, Asahi Kasei, Mitsubishi Tanabe, Otsuka, Medical Review, and Shiseido; research funding from MSD, Daiichi Sankyo, NanoCarrier, Eisai, Sysmex, Shionogi, IQVIA, Parexel International, Astellas, Mediscience, Sumitomo Dainippon A2 Healthcare, Ono, Taiho, Bayer, Yakult Honsha, and Sanofi. TS reports receiving honoraria from Chugai, Merck Serono, Bristol-Myers Squibb, Takeda, Yakult Honsha, Lilly, Bayer Yakuhin, Ono, Merck, Astellas Pharma, Taiho, and Nihonkayaku; consulting or advisory role from Bayer, Lilly, Ono, Takara Bio, Merck Serono, and Nihonkayaku; research funding from Yakult Honsha, Chugai, Ono, Sanofi, Lilly, Daiichi Sankyo, Merck Serono, Gilead Sciences, and Dainippon Sumitomo. TE reports receiving honoraria from Lilly, Taiho, BristolMyers Squibb Japan, Eisai, Daiichi Sankyo, Merck Serono, Chugai, Ono, Takeda, Bayer, and Sanofi; research funding from Daiichi Sankyo, Merck Serono, MSD, Novartis, Dainippon Sumitomo, Ono, Astellas Pharma, Lilly, Bayer, Nihonkayaku, Pfizer, and Bristol-Myers Squibb Japan. SN reports receiving honoraria from Taiho and Astrazeneca. AS reports receiving research funding from MSD, Eisai, Ono, Taiho, Takeda, and Bayer. AO reports receiving honoraria from Ono, BMS, Chugai, Taiho, Eisai, and Amgen; research funding from Bristol-Myers Squibb; immediate family member of Atsushi Ohtsu have been employed by Celgene. TY reports receiving research funding from Novartus, MSD, Sumitomo Dainippon, Chugai, Sanofi, Daiichi-Sankyo, Parexel, Ono, GlaxoSmithKline, and Boehringer Ingelheim.

\section{Author details}

1Department of Gastrointestinal Oncology, National Cancer Center Hospital East, Kashiwa, Japan. ${ }^{2}$ Department of Experimental Therapeutics, National Cancer Center Hospital East, 6-5-1 Kashiwanoha, Kashiwa, Chiba 277-8577, Japan. ${ }^{3}$ Translational Research Support Section, Translational Research Management Division, Clinical Research Support Office, National Cancer Center Hospital East, Kashiwa, Japan. ${ }^{4}$ Department of Pharmacy, National Cancer Center Hospital East, Kashiwa, Japan. ${ }^{5}$ Department of Clinical Oncology, Aichi Cancer Center Hospital, Nagoya, Japan. ${ }^{6}$ Department of Medical Oncology, Graduated School of Medicine, Kagawa University, Takamatsu, Japan.

Received: 24 August 2019 Accepted: 17 December 2019 Published online: 27 December 2019

\section{References}

1. International Agency for Research on Cancer: GLOBOCAN 2018. http://gco. iarc.fr/today/data/factsheets/cancers/10_8_9-Colorectum-fact-sheet.pdf. Accessed 02 July 2019.

2. Heinmann V, von Weikersthal LF, Decker T, Kiani A, Vehling-Kaiser U, AlBatran SE, et al. FOLFIRI plus cetuximab versus FOLFIRI plus bevacizumab as first-line treatment for patients with metastatic colorectal cancer (FIRE-3): a randomised, open-label, phase 3 trial. Lancet Oncol. 2014;15:1065-75.

3. Venook AP, Niedzwiecki D, Lenz HJ, Innocenti F, Fruth B, Meyerhardt JA, et al. Effect of first-line chemotherapy combined with cetuximab or bevacizumab on overall survival in patients with kras wild-type advanced or metastatic colorectal cancer. JAMA. 2017;317:2392-401.

4. Tabernero J, Yoshino T, Cohn AL, Obermannova R, Bodoky G, GarciaCarbonero $R$, et al. Ramucirumab versus placebo in combination with second-line FOLFIRI in patients with metastatic colorectal carcinoma that progressed during or after first-line therapy with bevacizumab, oxaliplatin, and a fluoropyrimidine (RAISE): a randomized, double-blind, multicenter, phase 3 study. Lancet Oncol. 2015:16:499-508.

5. Van Cutsem E, Tabernero J, Lakomy R, Prenen H, Prausová J, Macarulla T, et al. Addition of aflibercept to fluorouracil, leucovorin, and irinotecan improves survival in a phase III randomized trial in patients with metastatic colorectal cancer previously treated with an oxaliplatin-based regimen. J Clin Oncol. 2012;30:3499-506.

6. Grothey A, Van Cutsem E, Sobrero A, Siena S, Falcone A, Ychou M, et al. Regorafenib monotherapy for previously treated metastatic colorectal cancer (CORRECT): an international, multicenter, randomized, placebocontrolled, phase 3 trial. Lancet. 2013;318:303-12.

7. Mayer RJ, Van Cutsem E, Falcone A, Yoshino T, Garcia-Carbonero R, Mizunuma N, et al. Randomized trial of TAS-102 for refractory metastatic colorectal cancer. N Engl J Med. 2015;372:1909-19.

8. Yoshino T, Mizunuma N, Yamazaki K, Nishina T, Komatsu Y, Baba H, et al. TAS-102 monotherapy for pretreated metastatic colorectal cancer: a doubleblind, randomized, placebo-controlled phase 3 trial. Lancet Oncol. 2012;13: 993-1001. 
9. Xu J, Kim TW, Shen L, Sriuranpong V, Pan H, Xu R, et al. Results of a randomized, double-blind, placebo-controlled, phase III trial of trifluridine/ tipiracil (TAS-102) monotherapy in Asian patients with previously treated metastatic colorectal cancer: the TERRA study. J Clin Oncol. 2017:36:350-8.

10. Tsukihara H, Nakagawa F, Sakamoto K, Ishida K, Tanaka N, Okabe H, et al. Efficacy of combination chemotherapy using a novel oral chemotherapeutic agent, TAS-102, together with bevacizumab, cetuximab, or panitumumab on human colorectal cancer xenografts. Oncol Rep. 2015;33:2135-42.

11. Kuboki Y, Nishina T, Shinozaki E, Yamazaki K, Shitara K, Okamoto W, et al. TAS-102 plus bevacizumab for patients with metastatic colorectal cancer refractory to standard therapies (C-TASK FORCE): an investigator-initiated open-label, single-arm, multicenter, phase 1/2 study. Lancet Oncol. 2017;18: 1172-81.

12. Lesniewski-Kmak K, Moiseenko V, Saunders M, Wasan H, Argiles G, Borg C, et al. Phase II study evaluating trifluridine/tipiracil + bevacizumab and capecitabine + bevacizumab in first-line unresectable metastatic colorecta cancer (mCRC) patients who are non-eligible for intensive therapy (TASCO1): results of the primary analysis. Ann Oncol. 2018;29:mdy149.021.

13. Kotani D, Shitara K, Kawazoe A, Fukuoka S, Kuboki Y, Bando H, et al. Safety and efficacy of trifluridine/tipiracil monotherapy in clinical practice for patients with metastatic colorectal cancer: experience at a single institution. Clin Colorectal Cancer. 2016:15:e109-15.

14. Oki E, Makiyama A, Miyamoto Y, Kotaka M, Kawanaka H, Miwa K, et al. Trifluridine/tipiracil plus bevacizumab in elderly patients with previously untreated metastatic colorectal cancer (KSCC1602): A multicenter, phase II clinical trial. J Clin Oncol. 2019:37(suppl):abstr 3548.

15. Yoshino T, Oki E, Nozawa H, Eguchi-Nakajima T, Taniguchi H, Morita S, et al. Rationale and design of the TRUSTY study: a randomized, multicenter, open-label phase II/III study of trifluridine/tipiracil plus bevacizumab versus irinotecan, fluoropyrimidine plus bevacizumab as second-line treatment in patients with metastatic colorectal cancer progressive during following firstline oxaliplatin-based chemotherapy. ESMO Open. 2018;3:e000411.

16. Pfeiffer $P$, Yilmaz M, Möller S, Maltha L, Krogh M, Zitnjak D, et al. Randomized study evaluating trifluridine/tipiracil (TAS-102) versus + trifluridine/tipiracil + bevacizumab as last-line therapy in patients with chemorefractory unresectable metastatic colorectal cancer (mCRC). J Clin Oncol. 2019;37:637.

\section{Publisher's Note}

Springer Nature remains neutral with regard to jurisdictional claims in published maps and institutional affiliations.

Ready to submit your research? Choose BMC and benefit from:

- fast, convenient online submission

- thorough peer review by experienced researchers in your field

- rapid publication on acceptance

- support for research data, including large and complex data types

- gold Open Access which fosters wider collaboration and increased citations

- maximum visibility for your research: over $100 \mathrm{M}$ website views per year

At BMC, research is always in progress.

Learn more biomedcentral.com/submissions 